\title{
Three periodic solutions for a class of ordinary $p$-Hamiltonian systems
}

\section{Qiong Meng*}

\section{"Correspondence:}

mengqiong@qq.com

School of Mathematical Science,

Shanxi University, Taiyuan, Shanxi

030006, P.R. China

\begin{abstract}
We study the $p$-Hamiltonian systems $-\left(\left|u^{\prime}\right|^{p-2} u^{\prime}\right)^{\prime}+A(t)|u|^{p-2} u=\nabla F(t, u)+\lambda \nabla G(t, u)$, $u(0)-u(T)=u^{\prime}(0)-u^{\prime}(T)=0$. Three periodic solutions are obtained by using a three critical points theorem.
\end{abstract}

MSC: $34 \mathrm{~K} 13 ; 34 \mathrm{~B} 15 ; 58 \mathrm{E} 30$

Keywords: $p$-Hamiltonian systems; three periodic solutions; three critical points theorem

\section{Introduction}

Consider the $p$-Hamiltonian systems

$$
\left\{\begin{array}{l}
-\left(\left|u^{\prime}\right|^{p-2} u^{\prime}\right)^{\prime}+A(t)|u|^{p-2} u=\nabla F(t, u)+\lambda \nabla G(t, u), \\
u(0)-u(T)=u^{\prime}(0)-u^{\prime}(T)=0
\end{array}\right.
$$

where $p>1, T>0, \lambda \in(-\infty,+\infty), F:[0, T] \times \mathbf{R}^{N} \rightarrow \mathbf{R}$ is a function such that $F(\cdot, x)$ is continuous in $[0, T]$ for all $x \in \mathbf{R}^{N}$ and $F(\cdot, x)$ is a $C^{1}$-function in $\mathbf{R}^{N}$ for almost every $t \in[0, T]$, and $G:[0, T] \times \mathbf{R}^{N} \rightarrow \mathbf{R}$ is measurable in $[0, T]$ and $C^{1} \in \mathbf{R}^{N} . A=\left(a_{i j}(t)\right)_{N \times N}$ is symmetric, $A \in C\left([0, T], \mathbf{R}^{N \times N}\right)$, and there exists a positive constant $\lambda_{1}$ such that $\left(A(t)|x|^{p-2} x, x\right) \geq$ $\lambda_{1}^{p}|x|^{p}$ for all $x \in \mathbf{R}^{N}$ and $t \in[0, T]$, that is, $A(t)$ is positive definite for all $t \in[0, T]$.

In recent years, the three critical points theorem of Ricceri [1] has widely been used to solve differential equations; see [2-4] and references therein.

In [5], Li et al. have studied the three periodic solutions for $p$-Hamiltonian systems

$$
\left\{\begin{array}{l}
-\left(\left|u^{\prime}\right|^{p-2} u^{\prime}\right)^{\prime}+A(t)|u|^{p-2} u=\lambda \nabla F(t, u)+\mu \nabla G(t, u), \\
u(0)-u(T)=u^{\prime}(0)-u^{\prime}(T)=0
\end{array}\right.
$$

Their technical approach is based on two general three critical points theorems obtained by Averna and Bonanno [6] and Ricceri [4].

In [7], Shang and Zhang obtained three solutions for a perturbed Dirichlet boundary value problem involving the $p$-Laplacian by using the following Theorem A. In this paper, we generalize the results in [7] on problem (1.1).

Theorem A [1,7] Let $X$ be a separable and reflexive real Banach space, and let $\phi, \psi: X \rightarrow$ $\mathbf{R}$ be two continuously Gâteaux differentiable functionals. Assume that $\psi$ is sequentially

\section{楚 Springer}

(c) 2014 Meng; licensee Springer. This is an Open Access article distributed under the terms of the Creative Commons Attribution License (http://creativecommons.org/licenses/by/2.0), which permits unrestricted use, distribution, and reproduction in any medium, provided the original work is properly credited. 
weakly lower semicontinuous and even that $\phi$ is sequentially weakly continuous and odd, and that, for some $b>0$ and for each $\lambda \in[-b, b]$, the functional $\psi+\lambda \phi$ satisfies the PalaisSmale condition and

$$
\lim _{\|x\| \rightarrow \infty}[\psi(x)+\lambda \phi(x)]=+\infty
$$

Finally, assume that there exists $k>0$ such that

$$
\inf _{x \in X} \psi(x)<\inf _{|\phi(x)|<k} \psi(u)
$$

Then, for every $b>0$, there exist an open interval $\Lambda \subset[-b, b]$ and a positive real number $\sigma$, such that for every $\lambda \in \Lambda$, the equation

$$
\psi^{\prime}(x)+\lambda \phi^{\prime}(x)=0
$$

admits at least three solutions whose norms are smaller than $\sigma$.

\section{Proofs of theorems}

First, we give some notations and definitions. Let

$$
W_{T}^{1, p}=\left\{u:[0, T] \rightarrow \mathbf{R}^{N} \mid u \text { is absolutely continuous, } u(0)=u(T), u^{\prime} \in L^{p}\left(0, T ; \mathbf{R}^{N}\right)\right\}
$$

and is endowed with the norm

$$
\|u\|=\left(\int_{0}^{T}\left|u^{\prime}(t)\right|^{p} d t+\int_{0}^{T}\left(A(t)|u(t)|^{p-2} u(t), u(t)\right) d t\right)^{\frac{1}{p}} .
$$

Let $\varphi_{\lambda}: W_{T}^{1, p} \rightarrow \mathbf{R}$ be defined by the energy functional

$$
\varphi_{\lambda}(u)=\psi(u)+\lambda \phi(u)
$$

where $\psi(u)=\frac{1}{p}\|u\|^{p}-\int_{0}^{T} F(t, u(t)) d t, \phi(u)=\int_{0}^{T} G(t, u(t)) d t$.

Then $\varphi_{\lambda} \in C^{\prime}\left(W_{T}^{1, p}, \mathbf{R}\right)$ and one can check that

$$
\begin{aligned}
\left\langle\varphi_{\lambda}^{\prime}(u), v\right\rangle= & \int_{0}^{T}\left[\left(\left|u^{\prime}(t)\right|^{p-2} u^{\prime}(t), v^{\prime}(t)\right)-(\nabla F(t, u(t)), v(t))\right. \\
& -\lambda(\nabla G(t, u(t)), v(t))] d t
\end{aligned}
$$

for all $u, v \in W_{T}^{1, p}$. It is well known that the $T$-periodic solutions of problem (1.1) correspond to the critical points of $\varphi_{\lambda}$.

As $A(t)$ is positive definite for all $t \in[0, T]$, we have Lemma 2.1.

Lemma 2.1 For each $u \in W_{T}^{1, p}$,

$$
\lambda_{1}\|u\|_{L^{p}} \leq\|u\|,
$$

where $\|u\|_{L^{p}}=\int_{0}^{T}|u(t)|^{p} d t$. 
Theorem 2.1 Suppose that $F$ and $G$ satisfy the following conditions:

(H1) $\lim _{|x| \rightarrow \infty} \frac{|\nabla F(t, x)|}{\left.|x|\right|^{p-1}}=0$, for a.e. $t \in[0, T]$;

(H2) $\lim _{|x| \rightarrow 0} \frac{|\nabla F(t, x)|}{\left.|x|\right|^{p-1}}=0$, for a.e. $t \in[0, T]$;

(H3) $\lim _{|x| \rightarrow 0} \frac{F(t, x)}{\left.|x|\right|^{D}}=\infty$, for a.e. $t \in[0, T]$;

(H4) $|\nabla G(t, x)| \leq c\left(1+|x|^{q-1}\right), \forall x \in \mathbf{R}^{N}$, a.e. $t \in[0, T]$, for some $c>0$ and $1 \leq q<p$;

(H5) $F(t, \cdot)$ is even and $G(t, \cdot)$ is odd for a.e. $t \in[0, T]$.

Then, for every $b>0$, there exist an open interval $\Lambda \subset[-b, b]$ and a positive real number $\sigma$, such that for every $\lambda \in \Lambda$, problem (1.1) admits at least three solutions whose norms are smaller than $\sigma$.

Proof By (H1) and (H2), given $\varepsilon>0$, we may find a constant $C_{\varepsilon}>0$ such that

$$
\begin{aligned}
& |\nabla F(t, x)| \leq C_{\varepsilon}+\varepsilon|x|^{p-1}, \quad \text { for every } x \in \mathbf{R}^{N}, \text { a.e. } t \in[0, T], \\
& |F(t, x)| \leq C_{\varepsilon}+\frac{\varepsilon}{p}|x|^{p}, \quad \text { for every } x \in \mathbf{R}^{N} \text {, a.e. } t \in[0, T],
\end{aligned}
$$

and so the functional $\psi(u)$ is continuously Gâteaux differentiable functional and sequentially weakly continuous in the space $W_{T}^{1, p}$. Also, by (H4), we know $\phi(u)$ is sequentially weakly continuous. According to (H4), we get

$$
|G(t, x)| \leq c|x|+\frac{c}{p}|x|^{q}, \quad \text { for every } x \in \mathbf{R}^{N} \text {, a.e. } t \in[0, T] .
$$

For $\forall \lambda \in \mathbf{R}$, from the inequality (2.5) and (2.6), we deduce that

$$
\begin{aligned}
\psi(u)+\lambda \phi(u) & \geq \frac{1}{p}\|u\|^{p}-\int_{0}^{T}\left(C_{\varepsilon}+\frac{\varepsilon}{p}|u(t)|^{p}\right) d t-\lambda \int_{0}^{T}\left(c|u(t)|+\frac{c}{q}|u(t)|^{q}\right) d t \\
& \geq \frac{1}{p}\left(1-\frac{\varepsilon}{\lambda_{1}}\right)\|u\|^{p}-\frac{c \lambda}{q \lambda_{1}} T^{\frac{p-q}{q}}\|u\|^{q}-\frac{c \lambda}{\lambda_{1}} T^{\frac{p-1}{p}}\|u\|-\varepsilon T .
\end{aligned}
$$

Since $p>q, \varepsilon$ small enough, we have

$$
\lim _{\|u\| \rightarrow \infty}[\psi(u)+\lambda \phi(u)]=+\infty
$$

Now, we prove that $\varphi_{\lambda}$ satisfies the (PS) condition.

Suppose $\left\{u_{n}\right\}$ is a (PS) sequence of $\varphi_{\lambda}$, that is, there exists $C>0$ such that

$$
\varphi_{\lambda}\left(u_{n}\right) \rightarrow C, \quad \varphi_{\lambda}^{\prime}\left(u_{n}\right) \rightarrow 0 \quad \text { as } n \rightarrow \infty .
$$

Assume that $\left\|u_{n}\right\| \rightarrow \infty$. By (2.7), which contradicts $\varphi_{\lambda}\left(u_{n}\right) \rightarrow C$. Thus $\left\{u_{n}\right\}$ is bounded. We may assume that there exists $u_{0} \in W_{T}^{1, p}$ satisfying

$$
\begin{aligned}
& u_{n} \rightarrow u_{0}, \quad \text { weakly in } W_{T}^{1, p}, \quad u_{n} \rightarrow u_{0}, \quad \text { strongly in } L^{p}[0, T], \\
& u_{n}(x) \rightarrow u_{0}(x), \quad \text { a.e. } t \in[0, T] .
\end{aligned}
$$


Observe that

$$
\begin{aligned}
& \left\langle\varphi_{\lambda}^{\prime}\left(u_{n}\right), u_{n}-u_{0}\right\rangle \\
& =\int_{0}^{T}\left[\left(\left|u_{n}^{\prime}(t)\right|^{p-2} u_{n}^{\prime}(t), u_{n}^{\prime}(t)-u_{0}^{\prime}(t)\right)+\left(A(t)\left|u_{n}(t)\right|^{p-2} u_{n}(t), u_{n}(t)-u_{0}(t)\right)\right] d t \\
& \quad-\int_{0}^{T}\left(\left(\nabla F\left(t, u_{n}(t)\right), u_{n}(t)-u_{0}(t)\right)\right) d t \\
& \quad-\lambda \int_{0}^{T}\left(\nabla G\left(t, u_{n}(t)\right), u_{n}(t)-u_{0}(t)\right) d t .
\end{aligned}
$$

We already know that

$$
\left\langle\varphi_{\lambda}^{\prime}\left(u_{n}\right), u_{n}-u_{0}\right\rangle \rightarrow 0, \quad \text { as } n \rightarrow \infty
$$

By (2.4) and (H4) we have

$$
\begin{aligned}
& \int_{0}^{T}\left(\nabla F\left(t, u_{n}(t)\right), u_{n}(t)-u_{0}(t)\right) d t \rightarrow 0, \quad \text { as } n \rightarrow \infty, \\
& \int_{0}^{T}\left(\nabla G\left(t, u_{n}(t)\right), u_{n}(t)-u_{0}(t)\right) d t \rightarrow 0, \quad \text { as } n \rightarrow \infty .
\end{aligned}
$$

Using this, (2.8), and (2.9) we obtain

$$
\begin{aligned}
& \int_{0}^{T}\left[\left(\left|u_{n}^{\prime}(t)\right|^{p-2} u_{n}^{\prime}(t), u_{n}^{\prime}(t)-u_{0}^{\prime}(t)\right)+\left(A(t)\left|u_{n}(t)\right|^{p-2} u_{n}(t), u_{n}(t)-u_{0}(t)\right)\right] d t \rightarrow 0, \\
& \quad \text { as } n \rightarrow \infty
\end{aligned}
$$

This together with the weak convergence of $u_{n} \rightarrow u_{0}$ in $W_{T}^{1, p}$ implies that

$$
u_{n} \rightarrow u_{0}, \quad \text { strongly in } W_{T}^{1, p} \text {. }
$$

Hence, $\varphi_{\lambda}$ satisfies the (PS) condition. Next, we want to prove that

$$
\inf _{u \in W_{T}^{1, p}} \psi(u)<0 .
$$

Owing to the assumption (H3), we can find $\delta>0$, for $L>0$, such that

$$
|F(t, x)|>L|x|, \quad \text { for } 0<|x| \leq \delta \text {, and a.e. } t \in[0, T] \text {. }
$$

We choose a function $0 \neq v \in C_{0}^{\infty}([0, T])$, put $L>\|v\|^{p} /\left(p \int_{0}^{T}|v|^{p} d t\right)$, and we take $\varepsilon>0$ small. Then we obtain

$$
\begin{aligned}
\psi(\varepsilon v) & =\frac{1}{p}\|\varepsilon v\|^{p}-\int_{0}^{T} F(t, \varepsilon v(t)) d t \\
& \leq \frac{\varepsilon^{p}}{p}\|v\|^{p}-L \varepsilon^{p} \int_{0}^{T}|v(t)|^{p} d t<0 .
\end{aligned}
$$

Thus (2.10) holds. 
From $(\mathrm{H} 2), \forall \varepsilon>0, \exists \rho_{0}(\varepsilon)>0$ such that

$$
|\nabla F(t, x)| \leq \varepsilon|x|^{p-1}, \quad \text { if } 0<\rho=|x|<\rho_{0}(\varepsilon) .
$$

Thus

$$
\int_{0}^{T} F(t, u(t)) d t \leq \frac{\varepsilon}{p} \int_{0}^{T}|u(t)|^{p} d t \leq \frac{\varepsilon}{p \lambda_{1}}\|u\|^{p} .
$$

Choose $\varepsilon=\lambda_{1} / 2$, one has

$$
\begin{aligned}
\psi(u) & =\frac{1}{p}\|u\|^{p}-\frac{\varepsilon}{p \lambda_{1}}\|u\|^{p} \\
& =\frac{1}{2 p}\|u\|^{p}>0 .
\end{aligned}
$$

Hence, there exists $k>0$ such that

$$
\inf _{|\phi(u)|<k} \psi(u)=0
$$

So we have

$$
\inf _{u \in W_{T}^{1, p}} \psi(u)<\inf _{|\phi(u)|<k} \psi(u)
$$

The condition (H5) implies $\psi$ is even and $\phi$ is odd. All the assumptions of Theorem A are verified. Thus, for every $b>0$ there exist an open interval $\Lambda \subset[-b, b]$ and a positive real number $\sigma$, such that for every $\lambda \in \Lambda$, problem (1.1) admits at least three weak solutions in $W_{T}^{1, p}$ whose norms are smaller than $\sigma$.

Theorem 2.2 If F and G satisfy assumptions (H1)-(H2), (H4)-(H5), and the following condition $\left(\mathrm{H} 3^{\prime}\right)$ :

$\left(\mathrm{H}^{\prime}\right)$ there is a constant $B_{1}=\sup \left\{1 / \int_{0}^{T}|u(t)|^{p} d t:\|u\|=1\right\}, B_{2} \geq 0$, such that

$$
F(t, x) \geq 2 B_{1} \frac{|x|^{p}}{p}-B_{2}, \quad \text { for } x \in \mathbf{R}^{N} \text {, a.e. } t \in[0, T]
$$

Then, for every $b>0$, there exist an open interval $\Lambda \subset[-b, b]$ and a positive real number $\sigma$, such that for every $\lambda \in \Lambda$, problem (1.1) admits at least three solutions whose norms are smaller than $\sigma$.

Proof The proof is similar to the one of Theorem 2.1. So we give only a sketch of it. By the proof of Theorem 2.1, the functional $\psi$ and $\phi$ are sequentially weakly lower semicontinuous and continuously Gâteaux differentiable in $W_{T}^{1, p}, \psi$ is even and $\phi$ is odd. For every $\lambda \in \mathbf{R}$, the functional $\psi+\lambda \phi$ satisfies the (PS) condition and

$$
\lim _{\|u\| \rightarrow \infty}(\psi+\lambda \phi)=+\infty
$$


To this end, we choose a function $v \in W_{T}^{1, p}$ with $\|v\|=1$. By condition (H3), a simple calculation shows that, as $s \rightarrow \infty$,

$$
\begin{aligned}
\psi(s v) & =\frac{1}{p}\|s v\|^{p}-\int_{0}^{T} F(t, s v(t)) d t \\
& \leq \frac{s^{p}}{p}\|v\|^{p}-2 \frac{s^{p} B_{1}}{p} \int_{0}^{T}|v(t)|^{p} d t+B_{2} T \\
& \leq-\frac{s^{p}}{p}+B_{2} T \rightarrow-\infty .
\end{aligned}
$$

Then (2.11) implies that $\psi(s v)<0$ for $s>0$ large enough. So, we choose large enough, $s_{0}>0$, let $u_{1}=s_{0} v$, such that $\psi\left(u_{1}\right)<0$. Thus, we get

$$
\inf _{u \in W_{T}^{1, p}} \psi(u)<0
$$

By the proof of Theorem 2.1 we know that there exists $k>0$, such that

$$
\inf _{u \in W_{T}^{1, p}} \psi(u)<\inf _{|\phi(u)|<k} \psi(u)
$$

According to Theorem A, for every $b>0$ there exist an open interval $\Lambda \subset[-b, b]$ and a positive real number $\sigma$, such that for every $\lambda \in \Lambda$, problem (1.1) admits at least three weak solutions in $W_{T}^{1, p}$ whose norms are smaller than $\sigma$.

\section{Competing interests}

The author declares that they have no competing interests.

\section{Acknowledgements}

Supported by the Natural Science Foundation of Shanxi Province (No. 2012011004-1) of China.

Received: 17 December 2013 Accepted: 3 June 2014 Published online: 11 July 2014

\section{References}

1. Bonanno, G: Some remarks on a three critical points theorem. Nonlinear Anal. 54, 651-665 (2003)

2. Afrouzi, GA, Heidarkhani, S: Three solutions for a Dirichlet boundary value problem involving the $p$-Laplacian. Nonlinear Anal. 66, 2281-2288 (2007)

3. Ricceri, B: On a three critical points theorem. Arch. Math. 75, 220-226 (2000)

4. Ricceri, B: A three critical points theorem revisited. Nonlinear Anal. 70, 3084-3089 (2009)

5. Li, C, Ou, Z-Q, Tang, C: Three periodic solutions for $p$-Hamiltonian systems. Nonlinear Anal. 74, 1596-1606 (2011)

6. Averna, D, Bonanno, G: A three critical point theorems and its applications to the ordinary Dirichlet problem. Topol. Methods Nonlinear Anal. 22, 93-103 (2003)

7. Shang, $X$, Zhang, J: Three solutions for a perturbed Dirichlet boundary value problem involving the $p$-Laplacian. Nonlinear Anal. 72, 1417-1422 (2010) 